\title{
On the Extension of Certain Maps with Values in Spheres
}

by

\author{
Carlos BIASI, Alice K. M. LIBARDI, Pedro L. Q. PERGHER \\ and Stanisław SPIEŻ
}

Presented by Czesław BESSAGA

Summary. Let $E$ be an oriented, smooth and closed $m$-dimensional manifold with $m \geq 2$ and $V \subset E$ an oriented, connected, smooth and closed $(m-2)$-dimensional submanifold which is homologous to zero in $E$. Let $S^{n-2} \subset S^{n}$ be the standard inclusion, where $S^{n}$ is the $n$-sphere and $n \geq 3$. We prove the following extension result: if $h: V \rightarrow S^{n-2}$ is a smooth map, then $h$ extends to a smooth map $g: E \rightarrow S^{n}$ transverse to $S^{n-2}$ and with $g^{-1}\left(S^{n-2}\right)=V$. Using this result, we give a new and simpler proof of a theorem of Carlos Biasi related to the ambiental bordism question, which asks whether, given a smooth closed $n$-dimensional manifold $E$ and a smooth closed $m$-dimensional submanifold $V \subset E$, one can find a compact smooth $(m+1)$-dimensional submanifold $W \subset E$ such that the boundary of $W$ is $V$.

1. Introduction. The extension problem is whether, given topological spaces $X, Y$, a subspace $A \subset X$ and a continuous map $f: A \rightarrow Y$, one can find a continuous map $g: X \rightarrow Y$ such that $g_{\mid A}=f$. For example, if $D^{n}$ is the unit $n$-disk, with boundary $\partial\left(D^{n}\right)=S^{n-1}=$ the unit $(n-1)$ sphere, then the identity map Id : $S^{n-1} \rightarrow S^{n-1}$ cannot be extended to a map $g: D^{n} \rightarrow S^{n-1}$, and this non-extension result has as a consequence the famous Brouwer fixed-point theorem, which asserts that each continuous map $g: D^{n} \rightarrow D^{n}$ has a fixed point. In fact, this is a particular case of a stronger non-extension result: let $M^{n}$ be any $n$-dimensional, connected and closed manifold and $W^{n+1}$ an $(n+1)$-dimensional compact manifold

2000 Mathematics Subject Classification: Primary 55S36; Secondary 55S35.

Key words and phrases: ambiental bordism, oriented cobordism group, Euler class, Alexander duality isomorphism, Lefschetz duality isomorphism, Thom class, Thom transversality theorem.

The second author was partially supported by FAPESP, and the third by CNPq and FAPESP. 
whose boundary is $M^{n}$. Then Id : $M^{n} \rightarrow M^{n}$ cannot be extended to a map $W^{n+1} \rightarrow M^{n}$. More generally, the same is valid if we replace Id : $M^{n} \rightarrow M^{n}$ by a map of closed manifolds, $g: M^{n} \rightarrow V^{n}$, which induces an isomorphism in homology, $g_{*}: H_{n}\left(M^{n}\right) \rightarrow H_{n}\left(V^{n}\right)$, with any coefficients; for example, if $g$ is a homotopy equivalence. Inspired by this setting, we prove the following extension result.

TheOREM 1. Let $E$ be an oriented, smooth and closed $m$-dimensional manifold, with $m \geq 2$, and $V \subset E$ an oriented, connected, smooth and closed $(m-2)$-dimensional submanifold which is homologous (with $\mathbb{Z}$-coefficients) to zero in $E$. Let $S^{n-2} \subset S^{n}$ be the standard inclusion, where $n \geq 3$. Then every smooth map $h: V \rightarrow S^{n-2}$ has a smooth extension $g: E \rightarrow S^{n}$ transverse to $S^{n-2}$ and with $g^{-1}\left(S^{n-2}\right)=V$.

Theorem 1 gives a method to attack the ambiental bordism question, which asks whether, given a smooth closed $n$-dimensional manifold $E$ and a smooth closed $m$-dimensional submanifold $V \subset E$, one can find a compact smooth $(m+1)$-dimensional submanifold $W \subset E$ such that the boundary of $W$ is $V$; in this case, we say that $V$ bounds in $E$. If $V=S^{m}$ and $E=$ $S^{m+2}$, such a $W$ is called a Seifert surface for the knot $S^{m} \rightarrow S^{m+2}$. Hirsch considered a related question in his old paper [3]; specifically, he showed that if $V$ is an $m$-dimensional connected closed and oriented manifold which bounds, then there exists an embedding of $V$ into $\mathbb{R}^{n}$ which is a boundary in $\mathbb{R}^{n}$ when $n \geq 2 m$. In [6], Sato showed that every connected, closed and oriented submanifold $V^{m} \subset S^{m+2}$ bounds in $S^{m+2}$. In [1], C. Biasi obtained the following result, which in particular gives Sato's result: denote by $i$ : $V \rightarrow E$ the inclusion map and suppose $E$ and $V$ are oriented. Suppose that $(V, i)$ bounds as an element of the oriented cobordism group $\Omega_{m}(E)$. Then $V$ bounds in $E$ in the following cases: (i) $n=m+2$; (ii) $m \leq 3$ and $n \geq m+2$; (iii) $m=4, n \geq 6$ and $n \neq 7$ (evidently, $[(V, i)]=0$ in $\Omega_{m}(E)$ is always a necessary condition for $V$ to be a boundary in $E$ ). Using Theorem 1, we give a new and simpler proof of case (i).

2. Proofs. Homology and cohomology will be understood with $\mathbb{Z}$-coefficients. To simplify notation, if $X \subset Y$ and $\alpha \in H_{r}(X)$, we use the same notation $\alpha \in H_{r}(Y)$ for the image of $\alpha$ under the homomorphism induced by the inclusion $X \rightarrow Y$. If $W$ is an $n$-dimensional, oriented and closed manifold, we will denote by $\mu_{W} \in H_{n}(W)$ its fundamental homology class.

To prove Theorem 1 , denote by $\eta \rightarrow V$ and $\nu \rightarrow S^{n-2}$ the normal bundles of $V$ in $E$ and $S^{n-2}$ in $S^{n}$, and by $D(\eta), D(\nu), S(\eta)$ and $S(\nu)$ the associated disk bundles and sphere bundles. The symbols $D_{P}, D_{L}$ and $D_{A}$ will be used to denote, respectively, the Poincaré, Lefschetz and Alexander duality isomorphisms, with the convention that the domains of these 
maps are the cohomology $\mathbb{Z}$-modules. Denote by $D(\eta)_{*} \subset D(\eta)$ the subset of non-zero vectors, and by $U_{\eta} \in H^{2}\left(D(\eta), D(\eta)_{*}\right)$ the Thom class of $\eta$. By excision, $U_{\eta}$ can be considered as lying in $H^{2}(E, E-V)$, and $D_{L}: H^{s}(D(\eta), S(\eta)) \cong H^{s}\left(D(\eta), D(\eta)_{*}\right) \rightarrow H_{m-s}(D(\eta))$ can be seen as an isomorphism $D_{L}: H^{s}(E, E-V) \rightarrow H_{m-s}(E)$; in this setting, the inclusion map $i: V \rightarrow E$ can be viewed as the zero section. Write $j: E \rightarrow(E, E-V)$ for the inclusion map. Let $e \in H^{2}(V)$ be the Euler class of $\eta$.

We assert that $j_{*} i_{*}\left(\mu_{V}\right)=D_{A}(e)$ in $H_{m-2}(E, E-V)$. In fact, a basic property of Thom classes (sometimes used as their definition) is that $U_{\eta}=$ $D_{L}^{-1} i_{*}\left(\mu_{V}\right)$ (see, for example, [2, Chapter 6, Section 11]). Also, the composite homomorphism

$$
D_{A}(j i)^{*} D_{L}^{-1}: H_{m-2}(E) \rightarrow H^{2}(E, E-V) \rightarrow H^{2}(V) \rightarrow H_{m-2}(E, E-V)
$$

coincides with $j_{*}: H_{m-2}(E) \rightarrow H_{m-2}(E, E-V)$; this follows from the fact that the duality isomorphisms are essentially the cap product with the fundamental homology classes. The Euler class $e$ is given by $e=(j i)^{*}\left(U_{\eta}\right)$, and thus $D_{A}(e)=D_{A}(j i)^{*} D_{L}^{-1} i_{*}\left(\mu_{V}\right)=j_{*} i_{*}\left(\mu_{V}\right)$, which shows the assertion.

Since by hypothesis $\mu_{V}=0$ in $H_{m-2}(E)$, we get $e=0$, and we assert that this implies that $\eta$ is a trivial vector bundle. In fact, it is well known that the 2-dimensional oriented vector bundles over $V$ are in one-to-one correspondence with the homotopy classes of maps from $V$ into a classifying space $B S O(2),[V, B S O(2)]$. A model for $B S O(2)$ is the complex projective space $\mathbb{C} P^{\infty}=\lim _{n} \mathbb{C} P^{n}$ (with the weak topology). $\mathbb{C} P^{\infty}$ is an Eilenberg-MacLane space of type $(\mathbb{Z}, 2)$, and so $\left[V, \mathbb{C} P^{\infty}\right]$ is in one-to-one correspondence with $H^{2}(V, \mathbb{Z})$; choosing a generator $\alpha \in H^{2}\left(\mathbb{C} P^{\infty}, \mathbb{Z}\right) \cong \mathbb{Z}$, this correspondence can be given by $[f] \in\left[V, \mathbb{C} P^{\infty}\right] \mapsto f^{*}(\alpha) \in H^{2}(V, \mathbb{Z})$. On the other hand, it is also well known that the Euler class of the oriented 2-dimensional universal vector bundle over $\mathbb{C} P^{\infty}$ (which is the complex canonical line bundle) is either $\alpha$ or $-\alpha$. If $f \in\left[V, \mathbb{C} P^{\infty}\right]$ classifies $\eta \rightarrow V$, then the naturality of the Euler classes show that, up to sign, $f^{*}(\alpha)$ is the Euler class $e$ of $\eta$. It follows that $f^{*}(\alpha)=0$ and thus $f$ is homotopic to a constant map, so that $\eta$ is a trivial bundle. This outline follows from bundle theory and the material of [2, Chapter 7, Sections 13 and 14]; alternatively, see [8, Part III].

Since $\eta$ and $\nu$ are trivial bundles, $P:=D(\eta)$ and $T:=D(\nu)$ are trivial disk (smooth) bundles over $V$ and $S^{n-2}$, respectively. Moreover, $P$ and $T$ can be considered as tubular neighbourhoods of $V$ in $E$ and $S^{n-2}$ in $S^{n}$, respectively. Set $M:=E-\operatorname{int}(P), A:=S(\eta)=\partial(M)=\partial(P), N:=$ $S^{n}-\operatorname{int}(T)$ and $B:=S(\nu)=\partial(N)=\partial(T)$. By Proposition 4.3 of [1], there exists a cross section $r: V \rightarrow A$ such that $r_{*}\left(\mu_{V}\right)=0$ in $H_{m-2}(M)$. Note that, since $S^{1}$ is a Lie group, any (smooth) bundle $X \rightarrow B$ with fibre $S^{1}$ has the following property: 
for any (smooth) sections $s_{1}, s_{2}: B \rightarrow X$, there exists a (smooth) bundle isomorphism $g: X \rightarrow X$ inducing the identity on $B$ and such that $s_{2}=g s_{1}$.

Consequently, there exists a (smooth) bundle isomorphism $g: A \rightarrow V \times$ $S^{1}$ such that $g(r(v))=(v, 1)$ for every $v \in V$, where $1 \in S^{1}$. Let $G$ : $P \rightarrow V \times D^{2}$ be a (smooth) bundle isomorphism such that $G_{\mid A}=g$ and $G(v)=(v, 0)$ for every $v \in V$, where 0 is the centre of $D^{2}$. We identify $T$ with $S^{n-2} \times D^{2}$ in the standard way; then $B=S^{n-2} \times S^{1}$ and $N=D^{n-1} \times S^{1}$, with $\partial\left(D^{n-1}\right)=S^{n-2}$. Let $H: V \times D^{2} \rightarrow S^{n-2} \times D^{2}=T$ be defined by $H(v, w)=(h(v), w)$. Then $H G: P \rightarrow T$ is transverse to $S^{n-2}$ and $(H G)^{-1}\left(S^{n-2}\right)=V$. Thus an extension of $f:=\left(H_{\mid V \times S^{1}}\right) g: A \rightarrow B$ to a smooth map $M \rightarrow N$ gives an extension as stated in Theorem 1. To obtain this extension, the first step is to find a continuous extension $M \rightarrow N$.

Since $N$ is an Eilenberg-MacLane space of type $(\mathbb{Z}, 1)$, a continuous extension $M \rightarrow N$ of $f$ exists if and only if $\delta(i f)^{*}(\theta)=0$ in $H^{2}(M, A)$, where $i: B \rightarrow N$ is the inclusion map, $\delta: H^{1}(A) \rightarrow H^{2}(M, A)$ is the coboundary homomorphism and $\theta$ is a generator of $H^{1}(N) \cong \mathbb{Z}$ (see [7, Theorem 12, p. 428]). The diagram

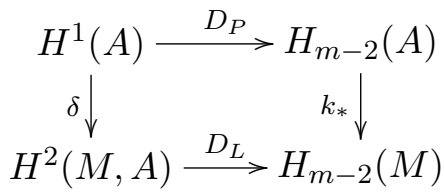

where $k: A \rightarrow M$ is the inclusion, is commutative (see [4, p. 379]). It follows that $\delta(i f)^{*}(\theta)=0$ if and only if $k_{*}\left(D_{P}(i f)^{*}(\theta)\right)=0$.

Now, we assert that $D_{P}(i f)^{*}(\theta)= \pm r_{*}\left(\mu_{V}\right)$. In fact, set $f^{\prime}=H_{\mid V \times S^{1}}$ : $V \times S^{1} \rightarrow B$. By the Künneth formula for cohomology, $\left(i f^{\prime}\right)^{*}(\theta)=u_{1} \times u_{2}$, where $u_{1} \in H^{0}(V)$ and $u_{2} \in H^{1}\left(S^{1}\right)$ are generators. Moreover, $\mu_{V \times S^{1}}=$ $\mu_{V} \times \mu_{S^{1}}$. By property 21 in [7, p. 255], with $\alpha$ a generator of $H_{0}\left(S^{1}\right)$, we obtain

$$
D_{P}\left(i f^{\prime}\right)^{*}(\theta)=\left(i f^{\prime}\right)^{*}(\theta) \cap \mu_{V \times S^{1}}=\left(u_{1} \cap \mu_{V}\right) \times\left(u_{2} \cap \mu_{S^{1}}\right)=\mu_{V} \times \alpha .
$$

Thus, since $g(r(v))=(v, 1)$, by the Künneth formula for homology, we deduce that $D_{P}\left(i f^{\prime}\right)^{*}(\theta)= \pm(g r)_{*}\left(\mu_{V}\right)$. It follows that $D_{P}(i f)^{*}(\theta)= \pm r_{*}\left(\mu_{V}\right)$, because $g: A \rightarrow V \times S^{1}$ is a homeomorphism and $f=f^{\prime} g$. Since $k_{*}\left(r_{*}\left(\mu_{V}\right)\right)$ $=0$, we obtain $\delta(i f)^{*}(\theta)=0$, and consequently we get the required continuous extension $M \rightarrow N$ of $f$. This extension can be slightly modified to give a map $M \rightarrow N$ which is smooth in a collar neighbourhood of $A$ in $M$. This last map can be approximated, without changing its values in a smaller collar neighbourhood of $A$ in $M$, by a smooth map $M \rightarrow N$. Together with $H G: P \rightarrow T$, this gives the desired smooth map $E \rightarrow S^{n}$ (for the approximation theorems for smooth maps used here and in the next corollary, see for example [5] and [9]). 
Corollary (C. Biasi, [1]). Let $E$ be an oriented, smooth and closed $m$ dimensional manifold with $m \geq 2, V \subset E$ an oriented, pathwise connected, smooth and closed $(m-2)$-dimensional submanifold, and $i: V \rightarrow E$ the inclusion map. If $(V, i)$ bounds as an element of the oriented cobordism group $\Omega_{m-2}(E)$, then $V$ bounds in $E$.

Proof. Consider $S^{3}$ as the one-point compactification $\left\{(x, y, z) \in \mathbb{R}^{3}\right.$ $\left.x^{2}+y^{2}+z^{2}<1\right\} \cup\{\infty\}$ and $S^{1}=\left\{(x, y, z) \in \mathbb{R}^{3} \mid x^{2}+y^{2}=1 / 4, z=0\right\} \subset S^{3}$, and let $h: V \rightarrow S^{1}$ be a constant map. Let $j: W^{m-1} \rightarrow E$ be a map that realizes the cobordism of $(V, i)$ in $\Omega_{m-2}(E)$ and let $k: V \rightarrow W^{m-1}$ be the inclusion map. Since $i=j k$ and $\mu_{V}=0$ in $H_{m-2}\left(W^{m-1}\right), \mu_{V}=0$ in $H_{m-2}(E)$. Evidently, the inclusion $S^{1} \rightarrow S^{3}$ has the properties of the standard inclusion $S^{n-2} \rightarrow S^{n}$ used in Theorem 1, hence this theorem applies to $h: V \rightarrow S^{1}$; as in its proof, denote by $P$ a closed tubular neigbourhood of $V$ in $E$ and by $T$ the closed tubular neighbourhood of $S^{1}$ in $S^{3}$ given by the product of $S^{1}$ and an orthogonal 2-disk of radius $1 / 4$. In the same way, set $M=E-\operatorname{int}(P), A=\partial(M)=\partial(P), N=S^{3}-\operatorname{int}(T)$ and $B=\partial(N)=\partial(T)$. As we have seen, $h: V \rightarrow S^{1}$ extends to a smooth map $F: E \rightarrow S^{3}$ transverse to $S^{1}$ and with $F^{-1}\left(S^{1}\right)=V$. Consider the Seifert surface $D \subset S^{3}$ for $S^{1}, D=\left\{(x, y, z) \in \mathbb{R}^{3} \mid x^{2}+y^{2} \leq 1 / 4, z=0\right\}$. Because of the construction of $F$ in the proof of Theorem 1, it is transverse to $D$ at every point in $F^{-1}(D) \cap P$. Then there exists an $\varepsilon$-approximation $F^{\prime}: E \rightarrow S^{3}$ for $F$ which is smooth, transverse to $D$ and with $F_{\mid P}^{\prime}=F_{\mid P}$. Then $F_{\mid P}^{\prime}{ }^{-1}\left(S^{1}\right)=V$, and for $\varepsilon$ sufficiently small the points of $E-P$ cannot be mapped by $F^{\prime}$ into $S^{1}$. The Thom transversality theorem then implies that $F^{\prime-1}(D)=W$ is an $(m-1)$-dimensional submanifold of $E$ whose boundary is $F^{\prime-1}\left(S^{1}\right)=V$, and the proof is finished.

\section{References}

[1] C. Biasi, On ambiental bordism, Pacific J. Math. 163 (1994), 73-80.

[2] G. E. Bredon, Topology and Geometry, Grad. Texts in Math. 139, Springer, New York, 1993.

[3] M. Hirsch, On embedding of bounding manifolds in euclidean space, Ann. of Math. 74 (1961), 494-497.

[4] W. S. Massey, A Basic Course in Algebraic Topology, Springer, New York, 1991.

[5] J. W. Milnor, Topology from the Differentiable Viewpoint, Univ. Press of Virginia, Charlottesville, 1965.

[6] N. Sato, Cobordism of semiboundary links, Topology Appl. 18 (1984), 225-234.

[7] E. H. Spanier, Algebraic Topology, McGraw-Hill, New York, 1966.

[8] N. E. Steenrod, The Topology of Fibre Bundles, Princeton Univ. Press, Princeton, NJ, 1951. 
[9] R. Thom, Quelques propriétés globales des variétés différentiables, Comment. Math. Helv. 28 (1954), 17-86.

Carlos Biasi

Departamento de Matemática

ICMC-USP - Campus de São Carlos

Caixa Postal 668

São Carlos, SP 13560-970, Brazil

E-mail: biasi@icmc.usp.br

Pedro L. Q. Pergher

Departamento de Matemática

Universidade Federal de São Carlos

Caixa Postal 676

São Carlos, SP 13565-905, Brazil

E-mail: pergher@dm.ufscar.br
Alice K. M. Libardi

Departamento de Matemática IGCE-UNESP - Campus de Rio Claro Rio Claro, SP 13506-700, Brazil E-mail: alicekml@rc.unesp.br

Stanisław Spież Institute of Mathematics Polish Academy of Sciences Śniadeckich 8, P.O. Box 21 00-956 Warszawa, Poland E-mail: spiez@impan.gov.pl

Received January 11, 2008;

received in final form June 16, 2008 